\title{
Partial dynamical symmetry from energy density functionals
}

\author{
K. Nomura, ${ }^{1}$ N. Gavrielov, ${ }^{2, *}$ and A. Leviatan ${ }^{2}$ \\ ${ }^{1}$ Department of Physics, Faculty of Science, University of Zagreb, HR-10000 Zagreb, Croatia \\ ${ }^{2}$ Racah Institute of Physics, The Hebrew University, Jerusalem 91904, Israel
}

(Dated: October 20, 2021)

\begin{abstract}
We show that the notion of partial dynamical symmetry is robust and founded on a microscopic many-body theory of nuclei. Based on the universal energy density functional framework, a general quantal boson Hamiltonian is derived and shown to have essentially the same spectroscopic character as that predicted by the partial SU(3) symmetry. The principal conclusion holds in two representative classes of energy density functionals: nonrelativistic and relativistic. The analysis is illustrated in application to the axially-deformed nucleus ${ }^{168} \mathrm{Er}$.
\end{abstract}

\section{INTRODUCTION}

Symmetries play a central role in quantum many-body physics. Dynamical symmetry (DS) is a class of symmetry that appears universally in diverse systems including hadrons [1], nuclei [2], molecules [3] and atoms [4]. The DS occurs if the Hamiltonian of the system can be written in terms of Casimir operators of a chain of nested algebras. The Hamiltonian is then exactly solvable and the spectra and wave functions are completely specified by the irreducible representations (irreps) of the algebras in the chain. In real quantum systems, however, an exact DS rarely occurs. More often some states obey the patterns required by the symmetry, but others do not. This necessitates a certain degree of symmetry-breaking, a prominent case of which is partial dynamical symmetry (PDS) [5, 6]. Its basic idea is to relax the stringent conditions imposed by an exact DS so that solvability and/or good symmetry are retained by only a subset of states. Detailed studies employing bosonic and fermionic models based on spectrum generating algebras, have shown that PDSs account quite well for a wealth of spectroscopic data in various types of nuclei [5-16] and are relevant to related quantum phase transitions and shapecoexistence [17-21].

One drawback in the implementation of symmetrybased notions in composite systems is that they are, in most cases, used without much considerations of their microscopic basis, i.e., connection to more fundamental degrees of freedom. With that in mind, the role of an emergent $\operatorname{Sp}(3, \mathrm{R}) \mathrm{DS}$ in light nuclei has been recently demonstrated within a symplectic no-core configuration interaction framework built on realistic nucleon-nucleon potentials $[22,23]$. The existence of $\mathrm{SU}(3)$ quasi dynamical symmetry evolved into a DS by means of the similarity renormalization group, has been demonstrated for ${ }^{36}$ Ar using a universal $s d$ shell model interaction [24]. In the same context, and motivated by the empirical manifestations of partial symmetries in heavy nuclei, a microscopic justification of PDS is called for. In this work,

\footnotetext{
* Current address: Center for Theoretical Physics, Sloane Physics Laboratory, Yale University, New Haven, CT 06520-8120, USA
}

we present a first proof of principle that the notion of PDS is robust and founded on a microscopic quantum many-body theory of nuclei. This proposition is illustrated in the example of the Hamiltonian with partial $\mathrm{SU}(3)$ symmetry in the framework of the interacting boson model (IBM) [2], widely used for describing collective states in nuclei in terms of monopole $(s)$ and quadrupole $(d)$ bosons. Based on a fermionic mean-field framework, we determine microscopically a general boson Hamiltonian, which is then shown to produce eigenstates that are similar in structure to those of the PDS one. We apply the procedure to ${ }^{168} \mathrm{Er}$, a typical example of an axiallydeformed nucleus, in which the SU(3)-PDS was previously recognized on phenomenological grounds [6-8].

\section{IBM FRAMEWORK AND SU(3) PDS}

Shapes and symmetries in nuclei can be studied in the IBM framework with the following Hamiltonian [25]

$$
\hat{H}=h_{0} P_{0}^{\dagger}\left(\beta_{0}\right) P_{0}\left(\beta_{0}\right)+h_{2} P_{2}^{\dagger}\left(\beta_{0}\right) \cdot \tilde{P}_{2}\left(\beta_{0}\right)+\rho \hat{L} \cdot \hat{L} .
$$

Here $P_{0}^{\dagger}\left(\beta_{0}\right)=d^{\dagger} \cdot d^{\dagger}-\beta_{0}^{2}\left(s^{\dagger}\right)^{2}, P_{2 \mu}^{\dagger}\left(\beta_{0}\right)=\beta_{0} \sqrt{2} d_{\mu}^{\dagger} s^{\dagger}+$ $\sqrt{7}\left(d^{\dagger} d^{\dagger}\right)_{\mu}^{(2)}, \tilde{P}_{2 \mu}\left(\beta_{0}\right)=(-1)^{\mu} P_{2,-\mu}\left(\beta_{0}\right), \hat{L}$ the angular momentum operator and standard notation of angular momentum coupling is used. The first two terms in Eq. (1) comprise the most general intrinsic Hamiltonian appropriate for the dynamics of a prolate-deformed shape. This is consistent with the energy surface obtained by its expectation value in a coherent (intrinsic) state [26, 27], which has the form

$$
\begin{aligned}
E_{\mathrm{IBM}}(\tilde{\beta}, \gamma) & =N(N-1)\left(1+\tilde{\beta}^{2}\right)^{-2}\left[h_{0}\left(\tilde{\beta}^{2}-\beta_{0}^{2}\right)^{2}\right. \\
& \left.+2 h_{2} \tilde{\beta}^{2}\left(\tilde{\beta}^{2}-2 \beta_{0} \tilde{\beta} \cos 3 \gamma+\beta_{0}^{2}\right)\right]
\end{aligned}
$$

Here $(\tilde{\beta}, \gamma)$ are the quadrupole shape parameters in the IBM. For $h_{0}, h_{2} \geqslant 0$, the surface has a global minimum at $\left(\tilde{\beta}=\beta_{0}>0, \gamma=0^{\circ}\right)$, corresponding to a prolatedeformed equilibrium shape. The intrinsic Hamiltonian determines the band-structure. The last term in Eq. (1) determines the in-band rotational splitting and its contribution to the energy surface is $1 / N$ suppressed, hence negligible. For $\beta_{0}=\sqrt{2}$ and $h_{0}=h_{2}$, the Hamiltonian (1) 
Skyrme EDF

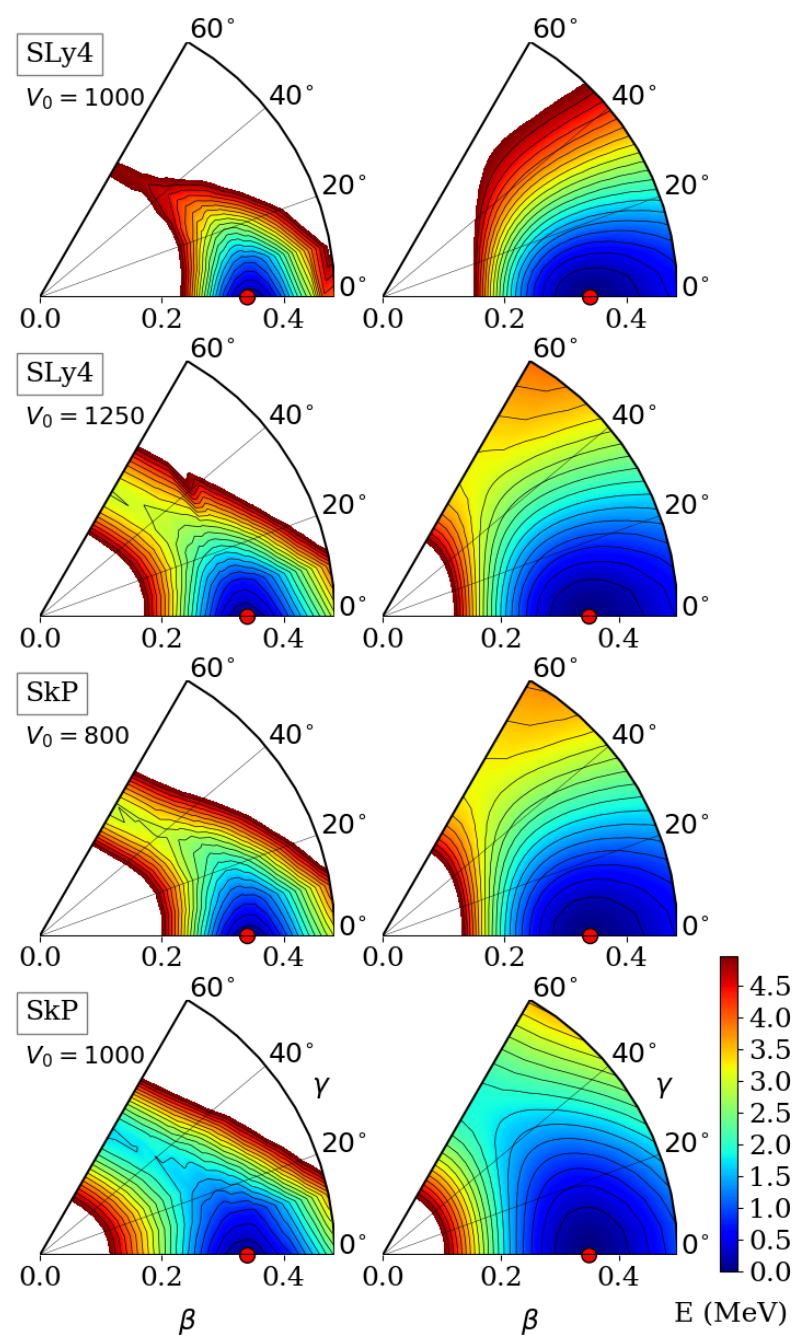

Relativistic EDF
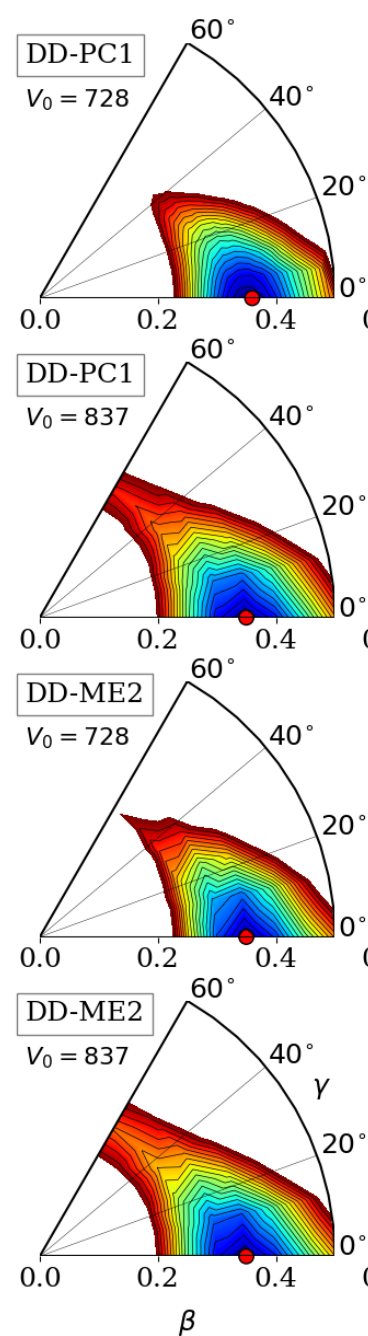

Mapped IBM

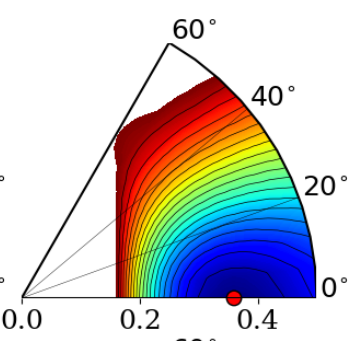

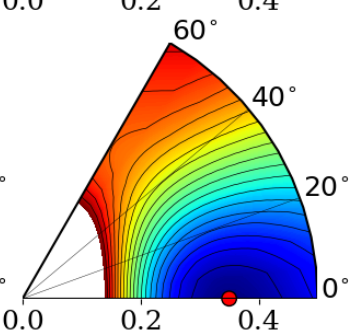

$0.0 \quad 60^{\circ}$
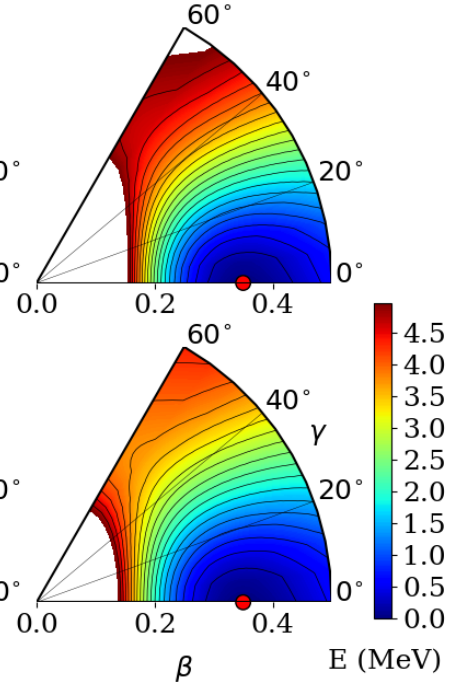

FIG. 1. SCMF energy surfaces in the $\beta-\gamma$ plane for ${ }^{168} \mathrm{Er}$, based on the nonrelativistic Skyrme SLy4 and SkP EDFs (first column) and the relativistic DD-PC1 and DD-ME2 EDFs (third column) with different values of pairing strengths $V_{0}$ in units of $\mathrm{MeVfm}^{3}$. The corresponding mapped IBM energy surfaces are plotted on the second and fourth columns. Contour spacing is $0.25 \mathrm{MeV}$, and the global minimum is indicated by a solid circle.

involves the Casimir operators of the algebras in the chain $\mathrm{U}(6) \supset \mathrm{SU}(3) \supset \mathrm{SO}(3)$, hence exhibits an $\mathrm{SU}(3)$ DS. The spectrum consists of $\mathrm{SU}(3)$ multiplets with the states $|[N](\lambda, \mu) K L\rangle$ specified by the total boson number $N$, the $\mathrm{SU}(3)$ irrep $(\lambda, \mu)$, the angular momentum $L$, and the label $K$ which corresponds to the projection of the angular momentum on the symmetry axis. The lowest multiplets have $(\lambda, \mu)=(2 N, 0)$ which contains the ground band $g(K=0)$, and $(\lambda, \mu)=(2 N-4,2)$ which contains both the $\beta(K=0)$ and $\gamma(K=2)$ bands. For $\beta_{0}=\sqrt{2}$ and $h_{0} \neq h_{2}$, the $\mathrm{SU}(3)$ symmetry is broken but selected bands maintain it [6]. In particular, the $(\lambda, \mu) \mathrm{SU}(3)$ classification remains intact for the ground and $\gamma$ bands, but the $\beta$ band is mixed. By definition, the resulting Hamiltonian has SU(3)-PDS. Previous studies in ${ }^{168} \mathrm{Er}$ employed the Hamiltonian (1) with $\beta_{0}=\sqrt{2}$ and remaining parameters determined from a fit [6]. The SU(3)-PDS predictions compared favorably with the empirical data for energies and E2 rates [6-8]. In what follows, we verify whether the features of SU(3)-PDS are realized by a microscopically-derived boson Hamiltonian.

\section{SCMF FRAMEWORK AND MAPPING PROCEDURE}

Among contemporary microscopic approaches, the nuclear energy density functional (EDF) framework allows for a reliable quantitative prediction of ground-state properties and collective excitations of nuclei over the entire region of the nuclear chart [28]. The basic implemen- 
tation of the EDF framework is in self-consistent meanfield (SCMF) methods, in which an EDF is constructed as a functional of one-body nucleon density matrices that correspond to a single product state. Both nonrelativistic $[29,30]$ and relativistic [31, 32] EDFs have been successfully applied to numerous studies of nuclear shaperelated phenomena and the resulting excitation modes and decay properties. In the present work, we consider these two representative classes of the EDF framework, so as to ensure the robustness of the results.

The starting point is a set of constrained SCMF calculations of an energy surface [33]. The constraints refer to those for mass quadrupole moments, which are associated with the polar deformation parameters $\beta$ and $\gamma$ [34]. $\beta$ is proportional to the intrinsic quadrupole moment and $\gamma$ specifies the departure from axiality. For $\gamma=0^{\circ}\left(\gamma=60^{\circ}\right)$ the nucleus is prolate (oblate) deformed, and intermediate values $0^{\circ}<\gamma<60^{\circ}$ correspond to triaxial shapes. The calculated SCMF energy surfaces $E_{\mathrm{SCMF}}(\beta, \gamma)$ for ${ }^{168} \mathrm{Er}$, are displayed on the first and third columns of Fig. 1. The former surfaces are obtained using the Hartree-Fock plus BCS model [35, 36] with the SLy4 [37] and SkP [38] parameterizations of the Skyrme EDF [39], while the latter using the relativistic Hartree-Bogoliubov model $[31,40]$ with the density-dependent point-coupling (DD-PC1) [41] and meson-exchange (DD-ME2) [42] functionals. Pairing correlations are taken into account by employing the density-dependent delta force and the separable pairing force of finite range [43] in the Skyrme and relativistic frameworks, respectively. For each EDF we consider several choices of pairing strength $V_{0}$. Specifically, for the nonrelativistic Skyrme EDFs: SLy4 with $V_{0}=1000$ and $1250 \mathrm{MeVfm}^{3}$, SkP with $V_{0}=800$ and $1000 \mathrm{MeVfm}^{3}$. A smooth cut-off [38] of $5 \mathrm{MeV}$ below and above the Fermi surface is invoked for these zero-range forces. The cut-off dependence of physical quantities was investigated in [44]. For the relativistic EDFs: both DDPC1 and DD-ME2 with $V_{0}=728$ and $837 \mathrm{MeVfm}^{3}$ (an increase by $15 \%$ ). The separable pairing force employed is practically identical to the finite-range Gogny interaction D1S, which does not need a cut-off and is known to give an excellent description of pairing over the periodic table. For nuclear matter, the pairing gap produced by this force is in good agreement with the results of abinitio calculations with the Bonn potential [45].

As is evident from Fig. 1, all adopted EDFs lead to energy surfaces accommodating a pronounced prolatedeformed global minimum $\left(\beta \approx 0.35, \gamma=0^{\circ}\right)$. The minimum tends to be less steep in $\beta$ and $\gamma$, for larger pairing strengths. This is anticipated because the quadrupole response function has two-quasiparticle energies in the denominator which increase with the pairing strength [33].

The IBM Hamiltonian of Eq. (1) is derived by the methods of [46-48]. The parameters $\left\{h_{0}, h_{2}, \beta_{0}\right\}$ are determined by mapping the microscopic energy surface $E_{\mathrm{SCMF}}(\beta, \gamma)$, obtained for a given $\mathrm{EDF}$, onto the corresponding IBM surface $E_{\mathrm{IBM}}(\beta, \gamma)$ of Eq. (2). The con-
TABLE I. Parameters $h_{0}, h_{2}, \rho$ (in $\mathrm{keV}$ ) and $\beta_{0}$, of the Hamiltonian (1) obtained from SCMF calculations based on nonrelativistic Skyrme SLy4 and SkP EDFs, and relativistic DD-PC1 and DD-ME2 EDFs, with pairing strengths $V_{0}$ (in $\mathrm{MeV} \mathrm{fm}{ }^{3}$ ). The corresponding parameters for SU(3)-PDS [6], are also shown. $E\left(2_{2}\right)$ and $E\left(0_{2}\right)$ are the calculated bandhead energies (in $\mathrm{keV}$ ) for the $\gamma$ and $\beta$ bands and $R=\frac{E\left(0_{2}\right)}{E\left(2_{2}\right)}$. For ${ }^{168} \mathrm{Er}, E\left(2_{2}\right)=821, E\left(0_{2}\right)=1217$ (in $\mathrm{keV}$ ) and $R=1.48$ [51].

\begin{tabular}{lccccc|ccc}
\hline \hline EDF & $V_{0}$ & $h_{0}$ & $h_{2}$ & $\rho$ & $\beta_{0}$ & $E\left(2_{2}\right)$ & $E\left(0_{2}\right)$ & $R$ \\
\hline SLy4 & 1000 & 10 & 5.3 & 11.8 & 1.59 & 1132 & 1911 & 1.68 \\
& 1250 & 10.4 & 4.0 & 12.3 & 1.39 & 809 & 1334 & 1.65 \\
SkP & 800 & 10.5 & 3.7 & 12.6 & 1.45 & 776 & 1306 & 1.68 \\
& 1000 & 30.6 & 4.4 & 12.2 & 0.99 & 672 & 1087 & 1.62 \\
DD-PC1 & 728 & 10.5 & 5.1 & 11.74 & 1.59 & 1092 & 1889 & 1.73 \\
& 837 & 9.8 & 4.4 & 11.73 & 1.51 & 925 & 1564 & 1.69 \\
DD-ME2 & 728 & 10.4 & 4.8 & 11.74 & 1.59 & 1032 & 1794 & 1.74 \\
& 837 & 9.9 & 4.2 & 11.73 & 1.50 & 883 & 1499 & 1.70 \\
\hline SU(3)-PDS & & 8.0 & 4.0 & 13.0 & $\sqrt{2}$ & 822 & 1220 & 1.48 \\
\hline \hline
\end{tabular}

dition $E_{\mathrm{SCMF}}(\beta, \gamma) \approx E_{\mathrm{IBM}}(\beta, \gamma)$ is imposed to ensure similar topology in the neighborhood of the global minimum. (The two surfaces are expressed in terms of $\beta$, since the IBM and SCMF deformations are related by $\tilde{\beta}=C \beta$, where the constant $C$ is determined by the mapping). The parameter $\rho$, Eq. (1), is obtained by equating the cranking moment of inertia in the IBM to the Thouless-Valatin value [49], the procedure discussed in detail in [48]. The mapped IBM energy surfaces, based on the nonrelativistic and relativistic EDFs, are shown on the second and fourth columns of Fig. 1, respectively. One clearly sees that the IBM and microscopic surfaces share common essential features near and up to a few $\mathrm{MeV}$ above the global minimum.

\section{EDF-BASED IBM HAMILTONIANS AND SPECTRA}

The values of the Hamiltonian parameters, derived microscopically from various EDFs, are given in Table I, along with the corresponding SU(3)-PDS parameters (obtained from a fit [6]). For SU(3)-PDS, $h_{0} / h_{2}=2$, while in most SCMF calculations, $1.9<h_{0} / h_{2}<2.8$, consistent with values obtained in global IBM fits in the rare-earth region [7]. The derived values of $\beta_{0}$ are close or slightly larger than the SU(3)-PDS value $\left(\beta_{0}=\sqrt{2} \approx 1.412\right)$. A notable exception are the parameters derived from the SkP EDF with pairing strength $V_{0}=1000 \mathrm{MeVfm}^{3}$, which exhibit pronounced large ratio $h_{0} / h_{2}=6.95$ and small $\beta_{0}=0.99$. This is a consequence of the fact that the corresponding SCMF energy surface for this case, shown in Fig. 1, is peculiarly soft in the $\gamma$ deformation, with a shallow local minimum on the oblate 

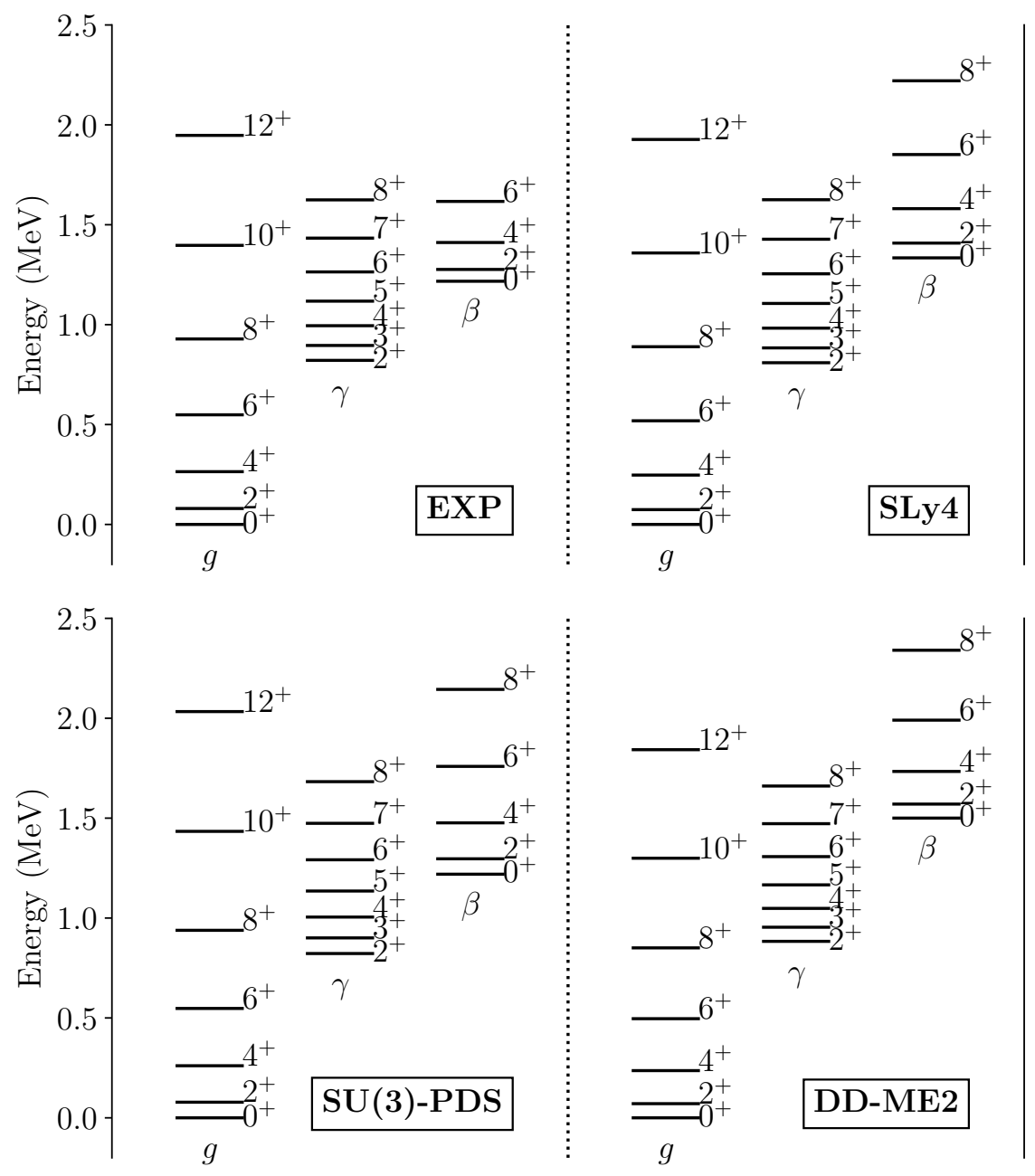

FIG. 2. Experimental [51] (EXP) and SU(3)-PDS [6] spectra for ${ }^{168}$ Er, compared with the spectra resulting from EDF-based IBM calculations for the Skyrme SLy4 EDF with pairing strength $V_{0}=1250 \mathrm{MeVfm}^{3}$, and for the relativistic EDF DD-ME2 with $V_{0}=837 \mathrm{MeVfm}^{3}$.

side. For any chosen EDF, a larger pairing strength results in a larger (smaller) value for $h_{0} / h_{2}\left(\beta_{0}\right)$.

Excitation spectra appropriate for ${ }^{168} \mathrm{Er}$ are obtained for each EDF by diagonalizing [50] the Hamiltonian (1) using the parameters in Table I and $N=16$. Typical spectra resulting from representative nonrelativistic and relativistic EDFs are displayed in Fig. 2. They satisfactorily conform with the calculated SU(3)-PDS spectrum which, in turn, agrees with experimental spectrum. The bandhead energies, $E\left(2_{2}\right)$ and $E\left(0_{2}\right)$ for the $\gamma$ and $\beta$ bands, and their ratios for the different cases, are listed in Table I. In general, the descriptions for the ground and $\gamma$ bands are stable with respect to different choices of EDFs. The description of the $\beta$-band is more casesensitive and all EDFs place $E\left(0_{2}\right)$ above the empirical and SU(3)-PDS values. The following observations are in order. (i) The relativistic EDFs generally result in higher $\beta$-band energies than the Skyrme EDFs. (ii) The increase of the pairing strength $\left(V_{0}\right)$ systematically de- creases the $\beta$-band energies. (iii) The SkP EDF with $V_{0}=1000 \mathrm{MeVfm}^{3}$, is the only case where both $E\left(2_{2}\right)$ and $E\left(0_{2}\right)$ are placed below the SU(3)-PDS and empirical values.

\section{SYMMETRY ANALYSIS}

Analysis of wave functions is a more sensitive measure to quantify the similarities and differences in structure between the EDF-based IBM Hamiltonians and SU(3)PDS. Fig. 3 shows the $\mathrm{SU}(3)(\lambda, \mu)$-decomposition for member states of the lowest bands in ${ }^{168} \mathrm{Er}$. For $\mathrm{SU}(3)$ PDS, the ground and $\gamma$ bands are pure with SU(3) character $(2 N, 0)$ and $(2 N-4,2)$, respectively, whereas the $\beta$ band contains a mixture of irreps: $(2 N-4,2) 87.5 \%$, $(2 N-6,0) 9.6 \%$, and $(2 N-8,4) 2.9 \%$, with $N=16$. Remarkably, for all nonrelativistic and relativistic EDFs considered (except SkP with pairing strength $V_{0}=1000$ 


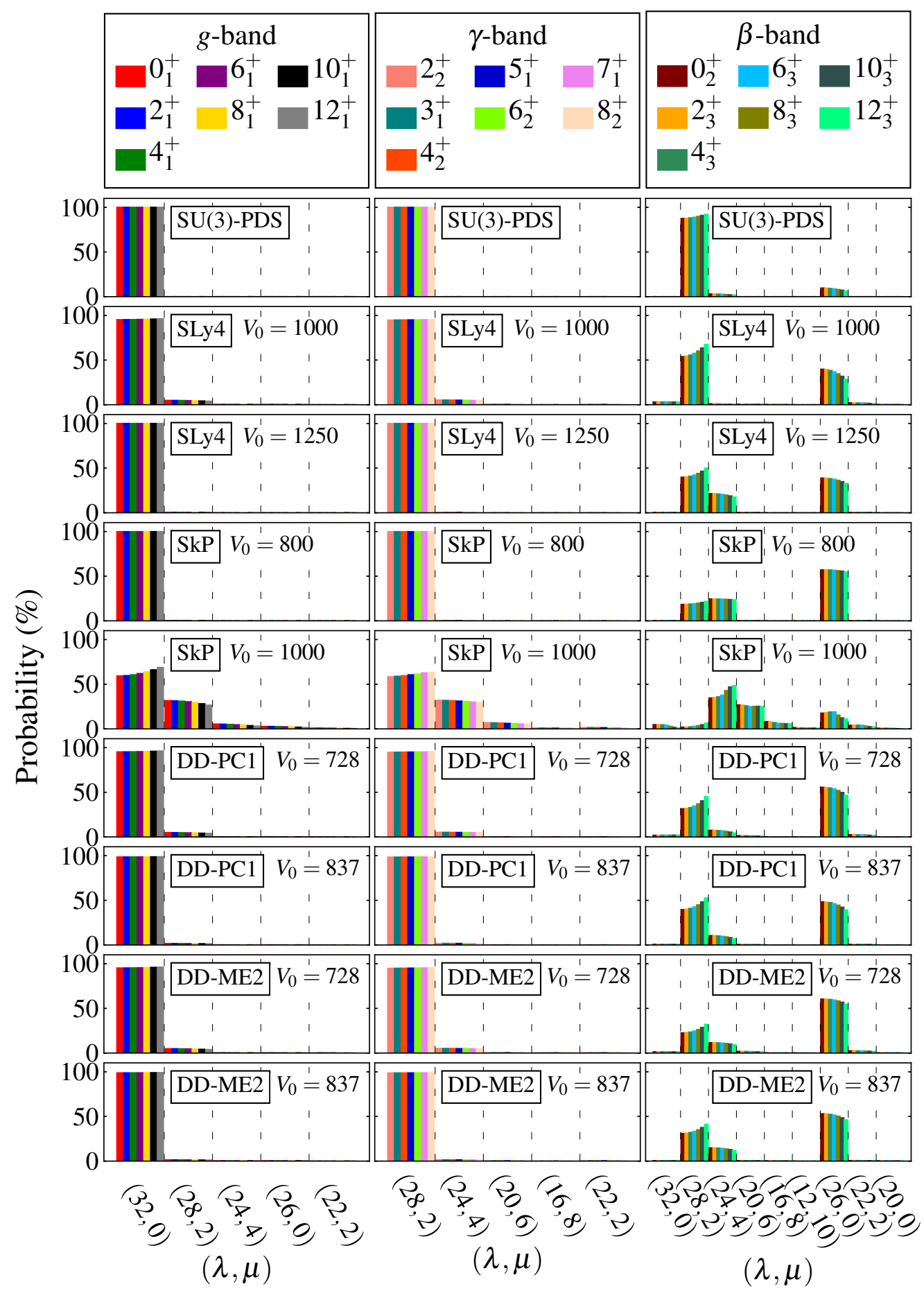

FIG. 3. $\mathrm{SU}(3)(\lambda, \mu)$-decomposition of states in the ground $(g), \gamma$ and $\beta$ bands, for the SU(3)-PDS and various EDF-based calculations. Shown are probabilities larger than $0.5 \%$. The histograms shown from left-to-right for each band, correspond to the $L_{i}$ states listed in the upper panels in the order top-to-bottom left-to-right.

$\mathrm{MeVfm}^{3}$ ), the mapped IBM Hamiltonians reproduce very well the SU(3)-PDS prediction of SU(3)-purity for the ground and $\gamma$ bands, with probability larger than $95 \%$. This clearly demonstrates the robustness of the PDS notion and its microscopic roots. The structure of the $\beta$ band is more sensitive to the choice of EDF. Its $\mathrm{SU}(3)$ mixing is governed by the values of the parameters $\beta_{0}$ and ratio $h_{0} / h_{2}$ which, in turn, reflect the different topology of the corresponding SCMF surfaces. Although the dominance of the $(2 N-4,2),(2 N-6,0)$, and $(2 N-8,4)$ irreps 
in the $\beta$ band is generally observed in all cases, their relative weights differ from those of SU(3)-PDS. This may indicate that additional degrees of freedom not included in the IBM (e.g., quasi particles) contribute to the structure of the $K=0_{2}$ band in ${ }^{168} \mathrm{Er}$. Again, the situation is different for the EDF SkP with $V_{0}=1000 \mathrm{MeVfm}^{3}$ for which the $\mathrm{SU}(3)$ decomposition exhibits large fragmentation. From all the EDFs considered, the SLy4 and SkP with $V_{0}=1250$ and $800 \mathrm{MeVfm}^{3}$, respectively, appear to yield spectral properties which are closest to the $\mathrm{SU}(3)$ PDS predictions for ${ }^{168} \mathrm{Er}(\mathrm{SU} / 3)$ purity for the ground and $\gamma$ bands with probability $99.8 \%$ ).

\section{CONCLUSIONS}

We have shown that the occurrence of partial dynamical symmetry (PDS) in nuclei can be justified from a microscopic point of view. By employing the constrained mean-field methods with choices of the universal energy density functionals and pairing interactions, in combination with symmetry analysis of the wave functions of the mapped IBM Hamiltonians, we arrived at an efficient procedure to test and explain the emergence of PDS in nuclei. An application to ${ }^{168} \mathrm{Er}$, has shown that the boson Hamiltonians derived from known EDFs in this region, produced eigenstates whose properties resemble those of SU(3)-PDS. The fact that these results are valid for both nonrelativistic and relativistic EDFs with several choices of pairing strengths, highlights the robustness of the PDS notion and its association with properties of the multi- nucleon dynamics in nuclei.

The results of the present investigation pave the way for a number of research avenues. (i) Exploring the microscopic origin of other types of PDSs, e.g., $\mathrm{SO}(6)$ PDS in $\gamma$-soft nuclei [12]. (ii) When a PDS is found to be manifested empirically in certain nuclei, it can be used to constrain, improve and optimize (e.g., choice of the pairing strength) a given EDF in that region. (iii) Exploiting the demonstrated linkage between the microscopic EDF framework and the algebraic PDS notion, to predict uncharted regions of exotic nuclei, awaiting to be explored, where partial symmetries can play a role.

\section{ACKNOWLEDGMENTS}

The work of K.N. is supported by the Tenure Track Pilot Programme of the Croatian Science Foundation and the École Polytechnique Fédérale de Lausanne, and the Project TTP-2018-07-3554 Exotic Nuclear Structure and Dynamics, with funds of the Croatian-Swiss Research Programme and also by the QuantiXLie Centre of Excellence, a project co-financed by the Croatian Government and European Union through the European Regional Development Fund - the Competitiveness and Cohesion Operational Programme (Grant KK.01.1.1.01.0004). The work of N.G. and A.L. is supported by the Israel Science Foundation Grant 586/16. N.G. acknowledges support by the Israel Academy of Sciences for a Postdoctoral Fellowship Program in Nuclear Physics.
[1] A. Bohm, Y. Néeman and A.O. Barut, eds., Dynamical Groups and Spectrum Generating Algebras, (World Scientific, Singapore, 1988).

[2] F. Iachello and A. Arima, The Interacting Boson Model (Cambridge University Press, Cambridge, 1987).

[3] F. Iachello and R.D. Levine, Algebraic Theory of Molecules (Oxford University Press, Oxford, UK, 1994),

[4] M. Kiselev, Y. Avishai and K. Kikoin, eds., Dynamical Symmetries for Nanostructures (Springer, Vienna, 2012).

[5] A. Leviatan, Prog. Part. Nucl. Phys. 66, 93 (2011).

[6] A. Leviatan, Phys. Rev. Lett. 77, 818 (1996).

[7] A. Leviatan and I. Sinai, Phys. Rev. C 60, 061301 (1999).

[8] R.F. Casten, R.B. Cakirli, K. Blaum and A. Couture, Phys. Rev. Lett. 113, 112501 (2014).

[9] R.F. Casten, J. Jolie, R.B. Cakirli and A. Couture, Phys. Rev. C 94, 061303(R) (2016).

[10] A. Couture, R.F. Casten and R.B. Cakirli, Phys. Rev. C 91, 014312 (2015).

[11] A. Leviatan, J.E. García-Ramos and P. Van Isacker, Phys. Rev. C 87, 021302(R) (2013).

[12] J.E. García-Ramos, A. Leviatan and P. Van Isacker, Phys. Rev. Lett. 102, 112502 (2009).

[13] P. Van Isacker, J. Jolie, T. Thomas and A. Leviatan, Phys. Rev. C 92, 011301(R) (2015).
[14] J. Escher and A. Leviatan, Phys. Rev. Lett. 84, 1866 (2000).

[15] D.J. Rowe and G. Rosensteel, Phys. Rev. Lett. 87, 172501 (2001).

[16] P. Van Isacker and S. Heinze, Ann. Phys. (NY) 349, 73 (2014).

[17] A. Leviatan, Phys. Rev. Lett. 98, 242502 (2007).

[18] M. Macek and A. Leviatan, Ann. Phys. (NY) 351, 302 (2014).

[19] A. Leviatan and D. Shapira, Phys. Rev. C 93, 051302(R) (2016).

[20] A. Leviatan and N. Gavrielov, Phys. Scr. 92, 114005 (2017).

[21] A. Leviatan, N. Gavrielov, J.E. García-Ramos and P. Van Isacker, Phys. Rev. C 98, 031302(R) (2018).

[22] T. Dytrych, K.D. Launey, J.P. Draayer, D.J. Rowe, J.L. Wood, G. Rosensteel, C. Bahri, D. Langr and R.B. Baker, Phys. Rev. Lett. 124, 042501 (2020).

[23] A.E. McCoy, M.A. Caprio, T. Dytrych, and P.J. Fasano Phys. Rev. Lett. 125, 102505 (2020).

[24] C.W. Johnson, Phys. Rev. Lett. 124, 172502 (2020).

[25] A. Leviatan, Ann. Phys. (NY) 179, 201 (1987).

[26] J.N. Ginocchio and M.W. Kirson, Phys. Rev. Lett. 44, 1744 (1980). 
[27] A.E.L. Dieperink, O. Scholten and F. Iachello, Phys. Rev. Lett. 44, 1747 (1980).

[28] N. Schunck, ed., Energy Density Functional Methods for Atomic Nuclei (IOP Publishing, Bristol, 2019).

[29] M. Bender, P.-H. Heenen and P.-G. Reinhard, Rev. Mod. Phys. 75, 121 (2003).

[30] L.M. Robledo, T.R. Rodríguez and R.R. RodríguezGuzmán, J. Phys. G 46, 013001 (2019).

[31] D. Vretenar and A.V. Afanasjev, G.A. Lalazissis and P. Ring, Phys. Rep. 409, 101 (2005).

[32] T. Nikšić, D. Vretenar and P. Ring, Prog. Part. Nucl. Phys. 66, 519 (2011).

[33] P. Ring and P. Schuck, The Nuclear Many-Body Problem, (Springer, Berlin, 1980).

[34] A. Bohr and B.R. Mottelson, Nuclear Structure, Vol. II (Benjamin, New York, USA, 1975).

[35] P. Bonche, H. Flocard and P.-H. Heenen, Comput. Phys. Commun. 171, 29 (2005).

[36] W. Ryssens, V. Hellemans, M. Bender and P.-H. Heenen Comput. Phys. Commun. 187, 175 (2015).

[37] E. Chabanat, P. Bonche, P. Haensel, J. Meyer and R. Schaeffer, Nucl. Phys. A 635, 231 (1998).

[38] J. Dobaczewski, H. Flocard and J. Treiner, Nucl. Phys. A 422, 103 (1984).

[39] T.H.R. Skyrme, Nucl. Phys. 9, 615 (1958).

[40] T. Nikšić, N. Paar, D. Vretenar and P. Ring, Comput. Phys. Commun. 185, 1808 (2014).
[41] T. Nikšić, D. Vretenar and P. Ring, Phys. Rev. C 78, 034318 (2008).

[42] G.A. Lalazissis, T. Nikšić, D. Vretenar and P. Ring, Phys. Rev. C 71, 024312 (2005).

[43] Y. Tian, Z.Y. Ma and P. Ring, Phys. Lett. B 676, 44 (2009).

[44] S. Karatzikos, A.V. Afanasjev, G.A. Lalazissis and P. Ring, Phys. Lett. B 689, 72 (2010).

[45] M. Serra, A. Rummel and P. Ring, Phys. Rev. C 65, 014304 (2001).

[46] K. Nomura, N. Shimizu and T. Otsuka, Phys. Rev. Lett. 101, 142501 (2008).

[47] K. Nomura, N. Shimizu and T. Otsuka, Phys. Rev. C 81, 044307 (2010).

[48] K. Nomura, T. Otsuka, N. Shimizu and L. Guo, Phys. Rev. C 83, 041302(R) (2011).

[49] J.-P. Delaroche, M. Girod, J. Libert, H. Goutte, S. Hilaire, S. Péru, N. Pillet and G.F. Bertsch, Phys. Rev. C 81, 014303 (2010).

[50] S. Heinze, (2008), computer program ArbModel (University of Cologne).

[51] Evaluated Nuclear Structure Data File (ENSDF) http://www.nndc.bnl.gov/ensdf. 